in XFZY on SBS group, parts of those decreased in SM on DEY group, which independent to that of modern medical therapy.

\section{GW23-e1392 CORONARY HEART DISEASE OF SYNDROME OF BLOOD STASIS AND DEFICIENCY OF BOTH VITAL ENERGY AND YIN THERAPY WITH PRESCRIPTION-SYNDROME CORRESPONDING: A RANDOMISED CONTROLLED TRIAL}

doi:10.1136/heartjnl-2012-302920c.17
Zhi-Hong Zhao, Jie Wang. Department of Cardiology, Guang Anmen Hospital, China Academy of Chinese Medical Sciences, Beijing 100053, China

\section{Objectives}

Results Compared coronary heart disease with two groups, syndrome of blood stasis (SBS) and deficiency of both vital energy and yin (DEY), and observed the effects of treated by prescription-syndrome corresponding or not.

A randomised, double-blinded, and placebo-controlled trial was applied. Eighty-eight patients, diagnosed as UA, were enrolled and equally randomised into two groups, SBS and DEY groups, treated with Shengmai Capsule (生脉胶囊, SM), or Xuefu Zhuyu Capsule (血府逐瘀胶囊, XFZY) on SBS group, DEY group, by prescriptionsyndrome corresponding or not, and administered with the corresponding medications respectively for 4 weeks. The clinical symptom, and its scores were recorded and compared among groups during and after the treatment. High sensitive reactive protein (hs$\mathrm{CRP}$ ), platelet aggregation (ADP), homocysteic acid (Hcy), matrix metalloproteinase-9 (MMP9), B-natriuretic peptide (BNP), platelet activating factor (PAF), myeloperoxidase (MPO), endothelin (ET) were examined in groups before and after the treatment.

Eighty-two patients completed the whole study. After the treatment, the total effective rates of the prescription-syndrome corresponding groups in ameliorating symptoms and signs, respectively, which were more obvious in XFZY on SBS group, and SM on DEY group than those of prescription-syndrome uncorresponding group. Also in the prescription-syndrome corresponding groups, chest distress, palpitation, short breath, spontaneous perspiration amendment significantly than those of prescription-syndrome uncorresponding groups. Before and after therapy, the serum markers, all have significantly decreased in XFZY on SBS group, only hs-CRP, Hcy, MPO have significantly decreased in SM on DEY group; in prescription-syndrome uncorresponding groups, there have no significantly changes in SBS group, and hs-CRP, Hcy, $\mathrm{MPO}, \mathrm{MMP9}$ have statistical significance in DEYgroup. After therapy, only in SBS group, there have statistical significance in prescription-syndrome corresponding or uncorresponding groups.

Conclusions Our CAD angina prescription-syndrome corresponding trial identified the on the basis of modern medical therapy, the differentiation of syndrome of CTM showed its distinct effect. Prescription-syndrome corresponding can improve the diagnostic of coronary heart disease, and decreased serum inflammation markers 